\title{
CARACTERISTIQUES BIOLOGIQUES DES CIVELLES (ANGUILLA ANGUILLA) LORS DE LA TRANSITION EN ESTUAIRE.
}

\author{
M.N. DE CASAMAJOR (1) (2), R. LECOMTE-FINIGER (2) ET P. PROUZET (1)
}

(1) Laboratoire Halieutique d'Aquitaine - Ifremer- Technopole Izarbel - Maison du Parc 64210 Bidart

(2) Laboratoire d'ichtyoécologie - EPHE-CNRS ESA8046 - Université de Perpignan - 66860 Perpignan cedex

\section{RÉSUMÉ}

Au cours de la saison de migration 1999/2000, de novembre à mars, un échantillonnage de civelles est réalisé dans le sud-ouest de la France. L'objectif de cet échantillonnage est de déterminer, à partir des caractéristiques biologiques (taille, masse, pigmentation, ADN/Poids sec, teneur en eau et otolithométrie) des individus capturés en zone côtière et estuarienne les modifications des paramètres biologiques des civelles entre ces deux secteurs géographiques. Un amaigrissement des individus est observé au cours de la traversée de l'estuaire en décembre ce qui n'est pas visible en février. Cependant, certains individus capturés en mer présentent une marque de transition en estuaire surtout en février. Le développement de la pigmentation suit les mêmes tendances que le développement des otolithes sauf pour les civelles au stade $\mathrm{V}_{\mathrm{B}}$. La définition du type d'otolithe est un indicateur plus précis que le développement pigmentaire pour déterminer le développement physiologique des individus. Les mesures des distances des différentes structures inscrites sur l'otolithe reflètent un passé larvaire similaire. Seul le rayon total de l'otolithe diffère entre les civelles capturées en mer et en estuaire en raison de la croissance de l'otolithe pendant la phase de transition. II apparaît à partir des données biochimiques, otolithométriques et pigmentaires un mélange important des flux de civelles en février.

Mots clés : civelle, otolithe, ADN/Poids sec, migration estuarienne, pigmentation

\section{BIOLOGICAL CHARACTERISTIC OF GLASS EELS (ANGUILLA ANGUILLA) AT THE TIME OF TRANSITION IN ESTUARY.}

\section{ABSTRACT}

During the season of migration 1999/2000, from November to March, a glass eels sampling was carried out in the south-west part of France. The objective of this sampling was to determine from the biological characteristics (size, mass, pigmentation, DNA/dry Weight, water content and otolithometry) the modifications of the biological parameters of glass eels between coastal zone and estuary. A slimming of the individuals was observed during the crossing of the estuary in December but not in February. However, some individuals captured at sea present a mark of transition in estuary particularly in February. The development of pigmentation follows the same tendencies as the development of otoliths except for the glass eels at the stage $V_{B}$. The definition of the type of otolith is an indicator more precise than the pigmentary development to determine the physiological state of the individuals. Measurements of the distances from the various structures registered on the otolith reflect a similar larval 
past. Only the total ray of the otolith differs between glass eels captured at sea and in estuary because of the growth of the otolith during the phase of transition. It appears starting from the data biochemical, otolithometric and pigmentary a significant mixture of flows of glass eels in February.

Key words: glass eels, otolith, ADN /dry Weight, estuarine migration, pigmentation

\section{INTRODUCTION}

La phase de transition du domaine marin au domaine fluvial est un événement important au cours du développement larvaire des civelles. Cette phase précise de la migration est peu connue d'un point de vue biologique. Lorsque les leptocéphales se métamorphosent en civelles aux abords du plateau continental, elles cessent de s'alimenter jusqu'au moment de la colonisation du milieu fluvial (LEE et LEE, 1989). Ainsi, pendant toute la transition en estuaire, les civelles sont en phase de jeûne et puisent dans leurs réserves énergétiques pour poursuivre la migration. L'activité métabolique réduite des civelles leur permet de jeûner sur une longue période malgré des conditions environnementales nécessitant une adaptation physiologique au changement de milieu (KAWAKAMI et al., 1999). Les fortes capacités osmotiques des civelles souvent évoquées (TOSI et al., 1988 ; CICCOTTI et al., 1993) leur permettraient également de limiter les pertes énergétiques pendant la traversée de l'estuaire.

Les caractéristiques morphométriques ont été étudiées sur de nombreux estuaires dont I'Adour (CHARLON et BLANC, 1982 ; DE CASAMAJOR et al., 2000). L'ensemble des travaux montre une diminution de la longueur et du poids moyen des civelles accompagnée d'un développement de la pigmentation au cours de la saison (BOETIUS et BOETIUS, 1989). Cependant, l'état physiologique des individus en migration n'a jamais été déterminé. Le seul indice ayant déjà été mesuré sur des civelles est le rapport ARN/ADN sur Anguilla japonica (KAWAKAMI et al., 1999). L'objectif de ce travail est d'utiliser un nouvel indice de condition à partir du rapport ADN/Poids sec sur la civelle pour déterminer un éventuel amaigrissement des individus au cours de la traversée de l'estuaire. Cet indice est utilisé sur des larves de poissons marins (BERGERON et al, 1991 ; BERGERON et al., 1997).

Ce changement de milieu s'inscrit sur l'otolithe, sous la forme d'une double marque en bordure externe (LECOMTE-FINIGER, 1992). Les conditions exactes de formation de cette marque (vitesse de dépôt, paramètres physico-chimiques) sont encore mal connues. En parallèle, la pigmentation des civelles transparentes débute. Les stades de pigmentation (ELIE et al., 1982) ne peuvent être considérés comme un indice du temps passé en estuaire car la température influence davantage le processus de mélanisation des cellules que le temps. L'étude des microstructures des otolithes de civelles est largement répandue sur différentes espèces du genre Anguilla (ARAl et al., 1997 ; ARAI et al., 1999 ; ARAl et al., 2000). L'objectif de ce travail est de mettre en parallèle la formation de la double marque de transition en estuaire et le développement de la pigmentation à partir de l'étude des lots de civelles capturées en mer et en estuaire.

\section{MATÉRIELS ET MÉTHODES}

\section{Cadre géographique}

Pour caractériser les civelles lors de la transition entre le plateau continental et l'estuaire, deux sites géographiques ont été échantillonnés; le premier en zone côtière et le deuxième en zone estuarienne (Figure1).

Les civelles d'estuaire proviennent de l'Adour, dans le sud-ouest de la France, et sont capturées au niveau d'Urt à $22 \mathrm{~km}$ de la limite transversale de la mer (Figure.1). 
Ce secteur est localisé en zone de propagation de la marée dynamique, mais la salinité est très faible pendant la période d'étude. Les civelles en migration sont donc déjà acclimatées à l'eau douce. Elles proviennent des campagnes expérimentales menées par le Laboratoire Halieutique d'Aquitaine de I'Ifremer et sont capturées aux tamis poussés en surface et en profondeur pendant le flot (Tableau I) (DE CASAMAJOR, 1998).

Les civelles de mer, proviennent de la plage de Moliets, en zone côtière (salinité autour de 30-35 $\mathrm{g.l}^{-1}$ ) à une trentaine de kilomètres au nord de l'embouchure de l'Adour (Figure1). Elles sont issues des captures professionnelles et prises par la technique de pêche dite "à la vague", c'est à dire avec un tamis tenu à la main dans la zone de déferlement des vagues pendant la marée montante. Ces individus vivent encore en eau salée.

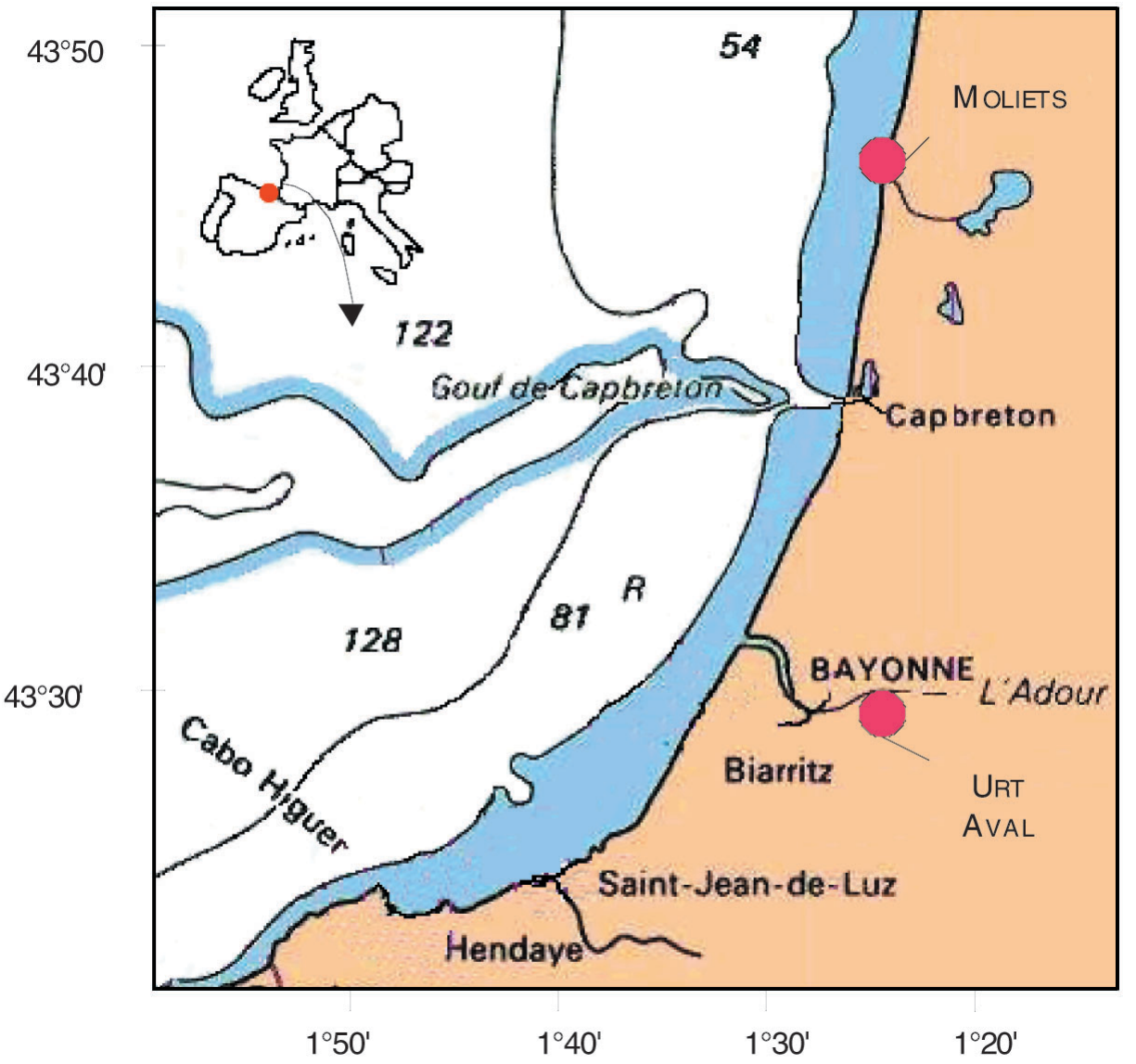

Figure 1

Localisation des sites de capture de civelles.

Figure 1

Sampling area of glass eels.

\section{Échantillonnages biologiques}

La principale saison de migration sur l'Adour se déroule entre novembre et mars. Pour caractériser le passage entre le milieu marin et le milieu continental, deux échantillonnages sont réalisés sur les deux sites, au mois de décembre et février, au moment de la nouvelle lune, soit 4 échantillons de 25 civelles (Tableau 1). Les paramètres environnementaux tels que le débit fluvial, le coefficient de marée, la 
température de la mer et la température de l'estuaire sont notés pour caractériser les conditions de migration des individus.

La masse (en g) la longueur (en $\mathrm{mm}$ ) et le stade pigmentaire (selon l'échelle d'ELIE et al. (1982)) sont répertoriés pour chaque individu. A leur arrivée en zone côtière, les civelles sont en majorité au stade $\mathrm{V}_{\mathrm{A}}$. La pigmentation se développe quand les civelles pénètrent en estuaire. Elles sont pour une large part au stade $\mathrm{V}_{\mathrm{B}}$. Ensuite, le processus de mélanisation se poursuit plus ou moins vite en fonction du temps de résidence en estuaire et de la température de l'eau. Les stades plus pigmentés ont été regroupés au sein du stade $\mathrm{VI}_{\mathrm{A}}$ car ils représentent un faible nombre d'individus dans l'échantillonnage en majorité au stade $\mathrm{VI}_{\mathrm{A} 0}$ et $\mathrm{VI}_{\mathrm{A} 1}$. Ainsi, trois stades de développement sont pris en compte $\left(\mathrm{V}_{\mathrm{A}}, \mathrm{V}_{\mathrm{B}}, \mathrm{VI}_{\mathrm{A}}\right)$.

\section{Tableau I}

Localisation géographique des échantillons récoltés.

( $n=$ nombre d'individus ; $C=$ coefficient de marée)

\section{Table I}

\section{Geographical location of samples \\ ( $n=$ number of glass eels ; $C=$ tide coefficient)}

\begin{tabular}{lccrrrrc}
\hline Date & $\begin{array}{c}\text { Localisation } \\
\text { cff.fig.1) }\end{array}$ & Origine & $\mathrm{N}$ & $\begin{array}{c}\text { Débit } \\
\mathrm{m}^{3} \cdot \mathrm{s}^{-1}\end{array}$ & $\mathrm{C}$ & $\begin{array}{c}\mathrm{T}^{\circ} \mathrm{C} \\
\mathrm{mer}\end{array}$ & $\begin{array}{c}\mathrm{T}^{\circ} \mathrm{c} \\
\text { estuaire }\end{array}$ \\
\hline $6 / 12 / 1999$ & Mer - Moliets & Pêche à la vague & 25 & 140 & 76 & 13,6 & 7,7 \\
\hline $9 / 12 / 1999$ & Adour - Urt & Pêche expérimentale & 25 & 142 & 78 & 13,7 & 7,4 \\
\hline $1 / 02 / 2000$ & Mer - Moliets & Pêche à la vague & 25 & 120 & 48 & 11,7 & 5,5 \\
\hline $8 / 02 / 2000$ & Adour - Urt & Pêche expérimentale & 25 & 157 & 87 & 12,6 & 9,2 \\
\hline
\end{tabular}

\section{Méthode de détermination de l'indice de condition}

Un indice biochimique d'amaigrissement est déterminé à partir du rapport ADN/Poids sec $\left(\mu \mathrm{g}_{\mathrm{mg}}{ }^{-1}\right)$ (BERGERON et al., 1991). Les larves sont lyophilisées pendant $24 \mathrm{~h}$ pour obtenir le poids sec avant d'être stockées dans des eppendorfs à $-80^{\circ} \mathrm{C}$ en attendant d'être broyées. L'homogénéisation de la civelle dans le liquide de broyage (héparine + PBS) est réalisée au mortier et au pilon, la méthode par "plotter" s'étant révélée inefficace pour l'obtention d'une solution homogène. Cet indice présente l'avantage sur le rapport ARN/ADN, d'une plus grande stabilité liée à l'instabilité des mesures de la molécule d'ARN. II est applicable à titre individuel, sur une petite quantité de matière sèche. La méthode fluorimétrique, employée pour déterminer la quantité d'ADN par larve a été proposée par LE PECQ et PAOLETTI (1966), puis modifiée et simplifiée par KARSTEN et WOLLENBERGER (1972). Les dosages des acides nucléiques sont réalisés suivant la méthode de KARSTEN et WOLLENBERGER (1977). L'ADN standard utilisé est le thymus de veau et les mesures d'ADN se font au spectrofluorimètre (longueurs d'ondes utilisées : excitation à $365 \mathrm{~nm}$ et émission à $590 \mathrm{~nm}$ ).

\section{Méthode de lecture des otolithes}

Les Sagittae sont prélevées sur des individus frais. Chaque otolithe est inclus dans une résine synthétique (type dentaire) et est poncé avec un papier fin (grain 1000). La zone poncée est polie, puis décalcifiée à l'EDTA (solution à $5 \%$ ) pour mettre en évidence les différentes structures inscrites dans l'otolithe. Des électronographies sont réalisées au Microscope Electronique à Balayage (MEB - Hitachi S520) après métallisation à l'or (couche de $20 \mathrm{~nm}$ ). Le grossissement le plus fréquemment utilisé est de 600 fois car il permet de mesurer les différentes phases de croissance depuis le 
nucleus jusqu'au bord externe de l'otolithe. Sur les 100 otolithes traités au cours de la saison de migration 1999/2000, 93 ont été interprétés.

L'interprétation de ces photographies permet une classification des otolithes et les mesures des différentes zones (Figure 2) suivant la description définie par LECOMTEFINIGER et YAHYAOUI (1989). Cette interprétation à partir des structures observées sur l'otolithe correspond au schéma de développement larvaire admis par la majorité des scientifiques travaillant sur le genre Anguilla (LECOMTE-FINIGER, 1994 ; DESAUNAY et GUERAULT, 1997, ARAI et al., 2000). Les otolithes sont classés suivant 3 types (Figure 2). Le type 1 correspond à l'absence de zone de transition "milieu marin et estuarien" à la périphérie de l'otolithe ; le type 2, à la présence d'une zone de transition en bordure d'otolithe. Le type 3 présente un développement de l'otolithe audelà de la marque qui traduit la pénétration dans les eaux estuariennes.

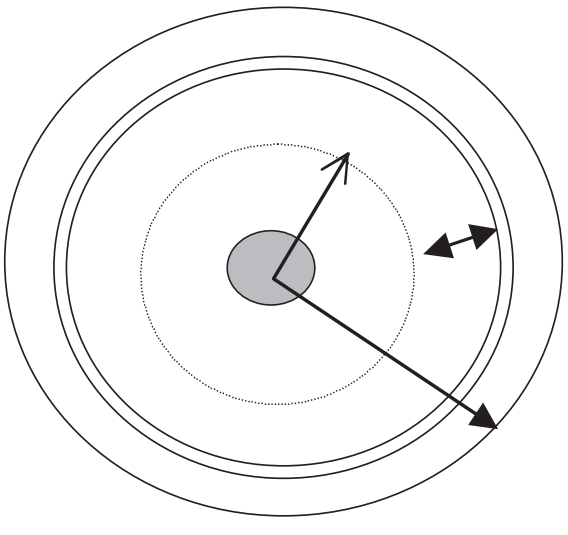

Marque de transition en estuaire

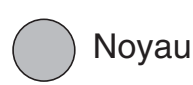
Noyau
Métamorphose Leptocéphale civelle

\section{Type 1}

Pas de marque de transition en estuaire

\section{Type 2}

Marque de transition en estuaire

en bordure d 'otolithe

\section{Type 3}

Début de croissance

après la marque de transition en estuaire

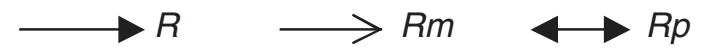

Figure 2

Classification des otolithes et mesures des différentes zones de croissance.

\section{Figure 2}

Otolith classification and measurements of the various zones of growth

Les mesures (en $\mu \mathrm{m}$ ) des principales zones de croissance inscrites sur l'otolithe sont décrites en figure 2. La distance $(R m)$ entre le nucleus et la zone de métamorphose de leptocéphale en civelle, correspond à la croissance de l'otolithe pendant la traversée de l'Océan Atlantique. Rt est la distance du nucleus à la double marque supposée matérialiser la zone de transition en estuaire. Le rayon total de l'otolithe $(R)$ représente sa croissance globale depuis l'éclosion jusqu'au bord. Cette mesure inclus l'épaisseur (é) de la zone de transition pour caractériser la traversée de l'estuaire. A partir de ces mesures, la distance $(R p)$ correspondant à la traversée du plateau continental durant la phase civelle est calculée.

\section{Traitements statistiques des données}

On utilise une relation d'allométrie de type $Y=a X^{b}$ pour étudier les variations conjointes de longueur et de poids entre les échantillons de mer et d'estuaire en 
décembre et en février. La comparaison du coefficient $b$ est réalisée par le test $t$ de Student après vérification des conditions d'application : indépendance des échantillons et normalité de la distribution. Ce test est également utilisé pour comparer les variations de mesures des structures des otolithes $(R, R m / R$ et $R p / R)$ entre les civelles de mer et d'estuaire en décembre et février. L'hypothèse d'égalité entre les deux échantillons est testée au risque $\propto=0,05$.

Les données ADN/Poids sec et teneurs en eau sont examinées, par classe de taille et stade pigmentaire, pour déterminer l'existence d'une différence significative liée à la pigmentation au cours du développement larvaire des civelles et celles liées aux conditions environnementales subies par les individus. Les données d'ADN/Poids sec et le pourcentage d'eau présentent une forte variabilité. De plus, l'utilisation d'un estimateur rapport ADN/Poids sec dont la distribution n'est pas forcément normale conduit à utiliser des tests non paramétriques pour remplir au mieux les conditions d'application et négliger les valeurs extrêmes induisant une forte variabilité. Pour les comparaisons des individus, capturés en mer et en estuaire, le test de rang de Wilcoxon est utilisé (WILCOXON, 1945). La condition d'indépendance des échantillons en vue de l'application de ces tests est vérifiée. L'hypothèse d'égalité entre les deux échantillons est testée au risque $\propto=0,05$.

\section{RÉSULTATS}

\section{Caractéristiques générales de l'échantillonnage}

La longueur et le poids moyens diminuent entre les échantillonnages de décembre et février (Tableau II). La pigmentation est plus développée en estuaire surtout en février où les individus au stade $\mathrm{V}_{\mathrm{B}}$ ne représentent plus que $58 \%$ (Tableau II). Le pourcentage d'individus non pigmentés est toujours supérieur en mer particulièrement au mois de décembre. A l'inverse, les individus fortement pigmentés $\left(\mathrm{VI}_{\mathrm{A}}\right)$ sont plus fréquents en estuaire et au mois de février sur les deux sites étudiés.

\section{Tableau II}

Caractéristiques morpho-métriques des civelles échantillonnées Moyenne (écart-type) et \% d'individus par stade pigmentaire.

\section{Table II}

Morpho-metric characteristic of glass eels sampled. Mean (standard deviation) and $\%$ of individuals for pigmentation stage.

\begin{tabular}{lcccccc}
\hline & Zone & Longueur & Poids & $\% \mathrm{~V}_{\mathrm{A}}$ & $\% \mathrm{~V}_{\mathrm{B}}$ & $\% \mathrm{~V}_{\mathrm{IA}}$ \\
\hline 06 décembre 1999 & Mer & $7,55(0,36)$ & $0,38(0,06)$ & 24 & 76 & 0 \\
\hline 09 décembre 1999 & Estuaire & $7,5(0,39)$ & $0,41(0,07)$ & 22 & 74 & 4 \\
\hline 01 février 2000 & Mer & $7,32(0,47)$ & $0,33(0,06)$ & 14 & 76 & 10 \\
\hline 08 février 2000 & Estuaire & $6,83(0,4)$ & $0,3(0,06)$ & 4 & 58 & 38 \\
\hline
\end{tabular}

La relation longueur-poids est significativement différente entre les deux secteurs géographiques (Student : décembre $t=2,3539 ; p=0,0206$ et février $t=-127,623 ; p \approx 0$ ). La différence est également significative entre les échantillons de décembre et de février (Student : estuaire $t=110,98 ; p \approx 0$ et mer $t=87,3 p \approx 0$ ).

\section{Indice Biochimique}

Une différence significative du rapport ADN/Poids sec et du pourcentage d'eau entre les civelles capturées en mer et en estuaire est observée en décembre. Ce n'est pas le cas en février (Tableau III). 


\section{Tableau III}

Résultats de l'analyse de rang réalisée sur le rapport ADN/Psec et \% d'eau sur les civelles au stade $V_{B}(A)$ tous stades pigmentaires confondus $(B)(\alpha=0,05)$

\section{Table III}

Results of Wilcoxon rank tests on percentage of water and DNA/Dry weight on glass eels $V_{B}(A)$ and all pigmentation stages (B) ) $(\alpha=0,05)$

A

\begin{tabular}{cccccc}
\hline & \multicolumn{2}{c}{ ADN /Poids Sec } & \multicolumn{2}{c}{$\% \mathrm{H} 2 \mathrm{O}$} & $\mathrm{H} 0$ \\
\hline & Wilcoxon & Proba & Wilcoxon & Proba & \\
\hline Décembre - mer-estuaire & 2,9586 & 0,0031 & 1,9977 & 0,0457 & Rejetée \\
\hline Février - mer-estuaire & 0,7408 & 0,4588 & 0,1443 & 0,8852 & Acceptée \\
\hline
\end{tabular}

B

\begin{tabular}{cccccc}
\hline VA - VB - VIA & \multicolumn{2}{c}{ ADN /Poids Sec } & \multicolumn{2}{c}{$\%$ H2O } & H0 \\
\hline Wilcoxon & Proba & Wilcoxon & Proba & & \\
\hline Décembre - mer-estuaire & $-2,4701$ & 0,0135 & 2,5303 & 0,0114 & Rejetée \\
\hline Février - mer-estuaire & 0,2426 & 0,8083 & $-0,3687$ & 0,7124 & Acceptée
\end{tabular}

Les médianes de l'indice ADN/poids sec par classe de taille montrent une diminution entre la mer et l'estuaire (Figure 3). L'analyse de rang montre que cette tendance n'est pas significative (Wilcoxon : décembre : $p=0,343$; février $p=0,686$ pour $\propto=0,05)$.

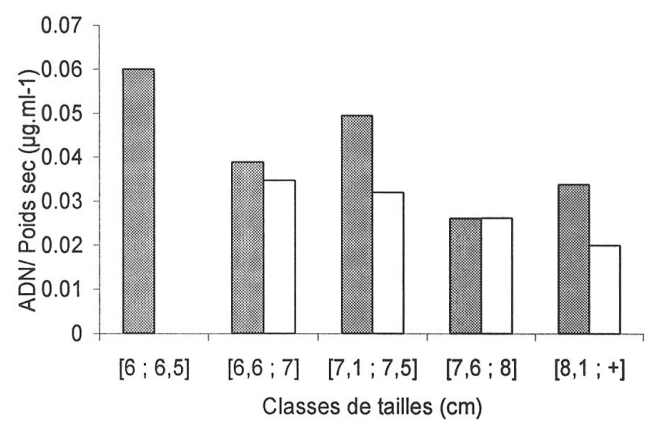

Décembre

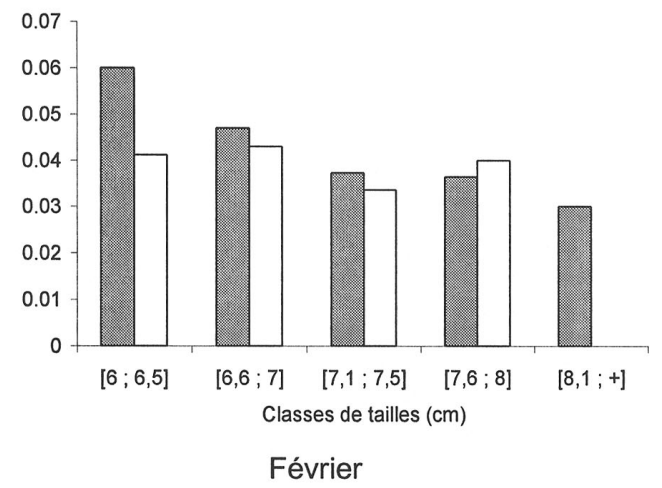

Estuaire/Estuary

Figure 3

Variations de la médiane du rapport ADN/Poids sec par classe de tailles de mer et d'estuaire pour les deux périodes d'échantillonnage.

\section{Figure 3}

Variations of the median of the DNA/Dry weight ratio by length classes at sea and estuary for the two sampling periods

Les médianes des teneurs en eau présentent des tendances similaires plus nuancées (Figure 4). Les fluctuations observées entre les échantillons de mer et d'estuaire ne sont pas significatives (Wilcoxon : décembre $p=0,685$; février $p=0,486$ pour $\propto=0,05$ ). 


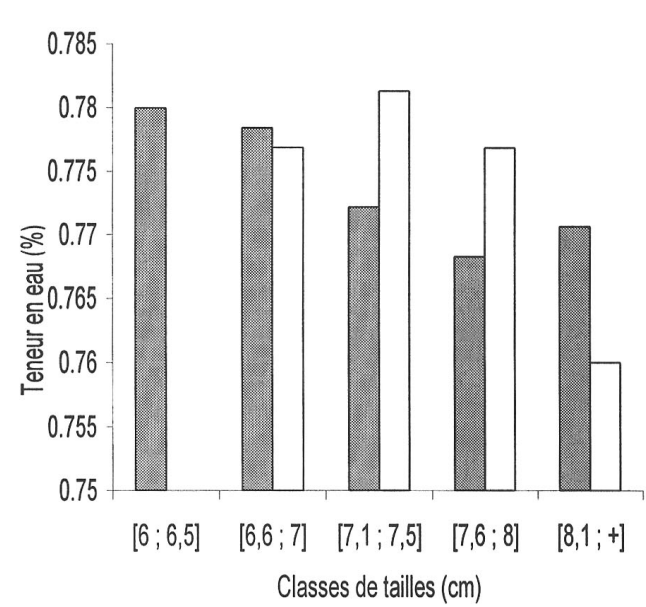

Décembre

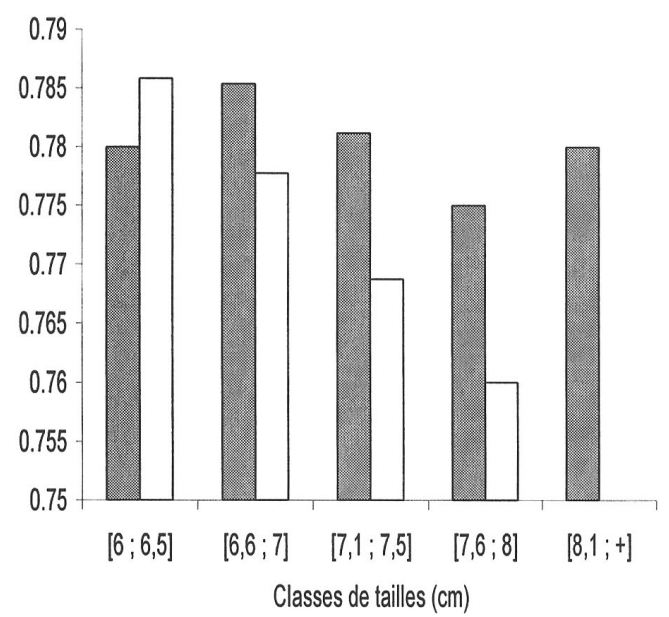

Février

Mer/Sea

Estuaire/Estuary

\section{Figure 4}

Variations de la médiane du pourcentage d'eau par classe de tailles en mer et en estuaire pour les deux périodes d'échantillonnage.

\section{Figure 4}

Variations of the median of the percentage of water by length classes at sea and estuary for the two sampling periods

\section{Otolithométrie -}

En décembre, les otolithes de type I sont majoritaires en mer et fortement représentés en estuaire. Leur proportion diminue au mois de février en mer comme en estuaire (Figure 5).

Les otolithes de type II sont peu nombreux dans les deux secteurs géographiques en décembre et leur proportion augmente en février dans les deux secteurs.

Les otolithes de type III sont absents de l'échantillonnage marin en décembre alors qu'ils représentent près de la moitié des effectifs en estuaire. En février, ils sont également présents en mer, mais en plus faible proportion qu'en estuaire.

En décembre, le stade $V_{A}$ est distribué de manière équivalente en mer et en estuaire. En février, son importance diminue tout en restant supérieure en mer. (Figure 6)

Les stades $\mathrm{V}_{\mathrm{B}}$ sont majoritaires. Ils sont en proportion équivalentes dans les deux secteurs géographiques en décembre. En février, ils sont mieux représentés en mer qu'en estuaire.

Les stades $\mathrm{VI}_{\mathrm{A}}$, sont absents en décembre dans l'échantillonnage en mer et faiblement représentés en estuaire. En février, ils sont peu nombreux en mer, mais représentent près de la moitié des individus capturés en estuaire. 


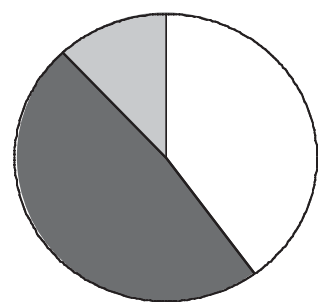

Décembre estuaire

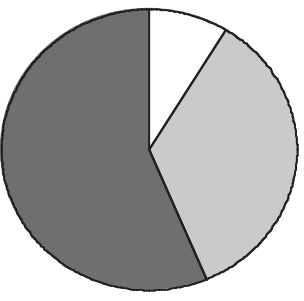

Février estuaire
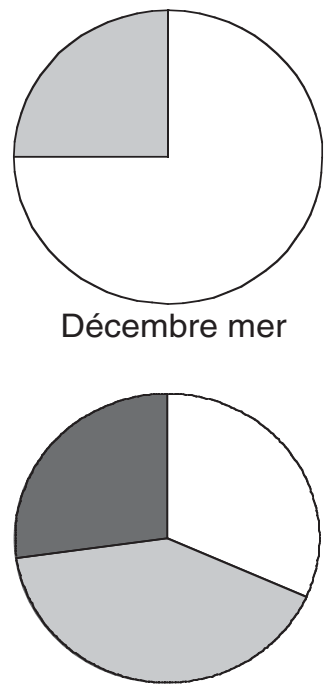

Février mer

Otolithes type 1

Otolithes type 2

Otolithes type 3

\section{Figure 5}

Proportion des différents types d'otilithe en fonction du mois et de l'origine des civelles capturées

\section{Figure 5}

Proportion of the various types of otolith according to the month and the origin of glass eels sampled

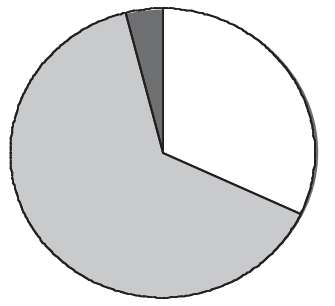

Décembre estuaire

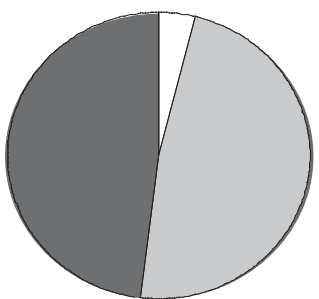

Février estuaire

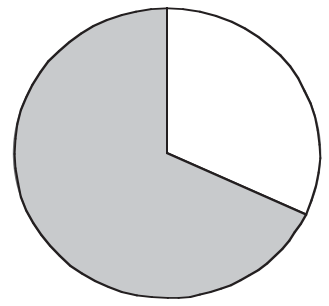

Décembre mer

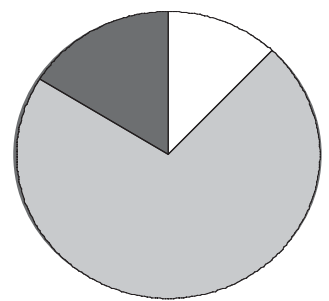

Février mer
Stade $V^{A}$
Stade $V^{B}$

Stade $\mathrm{VI}^{\mathrm{A}}$

Figure 6

Proportion des différents types d'otilithe en fonction du mois et de l'origine des civelles capturées

\section{Figure 6}

Proportion of the various types of otolith according to the month and the origin of glass eels sampled 
Il existe un lien entre la distribution des civelles en fonction du type d'otolithe et du stade pigmentaire (Tableau IV). Celles au stade $V_{A}$ ont des otolithes de type 1 alors que celles au stade $\mathrm{VI}_{\mathrm{A}}$ ont des otolithes de type 3 . Ces observations sont plus tranchées en mer qu'en estuaire. Par contre, on retrouve dans le type 2 tous les stades pigmentaires, particulièrement en estuaire.

\section{Tableau IV}

Répartition des otolithes en fonction de leur type et du stade pigmentaire des individus selon la saison et la localisation de l'échantillonnage.

\section{Table IV}

Distribution of otolith in regards to their type and pigmentation stage according to the season and location of the sampling area.

\begin{tabular}{c|ccc|ccc|ccc|crc}
\hline \multicolumn{8}{c|}{ ESTUAIRE } & \multicolumn{8}{c}{ MER } \\
\hline \multicolumn{9}{c|}{ Décembre } & \multicolumn{3}{c|}{ Février } & \multicolumn{3}{c}{ Décembre } & \multicolumn{3}{c}{ Février } \\
\hline Type & $\mathbf{1}$ & $\mathbf{2}$ & $\mathbf{3}$ & $\mathbf{1}$ & $\mathbf{2}$ & $\mathbf{3}$ & $\mathbf{1}$ & $\mathbf{2}$ & $\mathbf{3}$ & $\mathbf{1}$ & $\mathbf{2}$ & $\mathbf{3}$ \\
\hline $\mathrm{V}_{\mathrm{A}}$ & 8 & & & & & & 7 & & & 3 & \\
\hline $\mathrm{V}_{\mathrm{B}}$ & 2 & 11 & 3 & 2 & 5 & 6 & 10 & 6 & & 4 & 9 & 3 \\
\hline $\mathrm{VI}_{\mathrm{A}+}$ & & 1 & & & 3 & 7 & & & & & 3 \\
\hline
\end{tabular}

\section{Comparaison des mesures entre les civelles de mer et d'estuaire}

L'épaisseur (é) de la double marque inscrite en bordure d'otolithe pour les types 2 et 3 est constante autour de $5 \mu \mathrm{m}$ que ce soit pour les civelles prélevées en mer ou en estuaire. Cette mesure représente une proportion moyenne de $2,6 \%$ du rayon total de l'otolithe.

\section{Tableau V}

Comparaison des mesures réalisées sur les otolithes de civelles $(R, R m, R p)$ et leurs écarts standard ( ) en zone côtière et estuarienne.

\section{Table V}

Comparison of the measurements carried out on yhe otoliths of glas eels ( $R$, $\mathrm{Rm}, \mathrm{Rp}$ ) and their standard variations ( ) in coastal zone and estuary.

\begin{tabular}{lccc}
\hline & Phase leptocéphale $(R m)$ & Phase civelle $(R p)$ & Rayon total $(R)$ \\
\hline Décembre mer & $110,07(9,95)$ & $72,08(12,3)$ & $183,4(13,01)$ \\
\hline Décembre estuaire & $112,86(10,03)$ & $77,04(14,59)$ & $193,68(15,05)$ \\
\hline Février mer & $111,76(10,7)$ & $70,58(11,47)$ & $185,7(9,19)$ \\
\hline Février estuaire & $121,04(10,51)$ & $68,06(13,66)$ & $198,06(17,42)$ \\
\hline
\end{tabular}

Le rayon total $(R)$ des otolithes de civelles capturées en estuaire est significativement plus grand que celui des individus capturés en zone côtière en décembre comme en février (Tableau VI). Ceci traduit le développement de l'otolithe pendant la traversée de l'estuaire. 


\section{Tableau VI}

Comparaison des mesures otolithométriques effectuées sur les échantillons de mer et d'estuaire pour les mois de décembre et février par le test $\mathbf{t}$ de Student $(\propto=0,05)$.

\section{Table VI}

Comparison among otolith data sampled at sea and estuary in February and December with the Student t-test.

\begin{tabular}{lcccccc}
\hline & \multicolumn{3}{c}{ Décembre Mer-Estuaire } & \multicolumn{2}{c}{ Février Mer-Estuaire } \\
\hline & $t$ & $P$ & décision & $t$ & $P$ & Décision \\
\hline Rayon total $(R)$ & $-2,52$ & 0,015 & Rejetée & $-2,94$ & 0,005 & Rejetée \\
\hline Leptocéphale $(R m / R)$ & 1,08 & 0,285 & Acceptée & $-0,77$ & 0,45 & Acceptée \\
\hline Civelle $(R p / R)$ & -0.28 & 0,78 & Acceptée & 2,29 & 0,03 & Rejetée
\end{tabular}

La croissance de l'otolithe pendant les phases leptocéphale $(R m / R)$ et civelle $(R p / R)$ est identique entre les échantillonnages de mer et d'estuaire. (Tableau $\mathrm{V}$ et $\mathrm{VI}$ ). En outre, le rapport $(R p / R)$ est significativement différent en février entre les deux lots de civelles ce qui n'est pas le cas en décembre.

\section{DISCUSSION}

\section{Relation entre type d'otolithe et stade de pigmentation}

Le stade $\mathrm{V}_{\mathrm{B}}$ ne peut pas être considéré comme un marqueur du temps de résidence en estuaire, on le retrouve aussi bien en mer qu'en estuaire et les trois types d'otolithes sont présents au sein de ce stade. En mer, la majorité des civelles au stade $V_{A}$ ne présentent pas de double marque en bord d'otolithe. De même, en estuaire, les civelles pigmentées, regroupées en $\mathrm{VI}_{\mathrm{A}}$ présentent la double marque suivie d'une reprise de croissance témoignant de leur résidence en estuaire. En parallèle, le nombre de civelles pigmentées augmente entre décembre et février. De nombreux travaux (LECOMTE-FINIGER et YAHYAOUI, 1989 ; GUERAULT et al., 1992) montrent des tendances saisonnières identiques. DESAUNAY et al., (1996) montrent que l'otolithe et la pigmentation sont plus développés sur l'Adour que dans les autres estuaires français localisés plus au nord.

En conséquence, le stade pigmentaire est sur la côte sud Gascogne, un bon indicateur du stade de développement des otolithes sauf pour les stades $V_{B}$. La vitesse de pigmentation étant fortement dépendante de la température (STRUBBERG, 1913 ; UMEZAWA et TSUKAMOTO, 1991 ; ANTUNES et TESCH, 1997) la définition du type d'otolithe est plus pertinente pour définir le stade de développement des individus. II est admis que la double marque en bordure d'otolithe, différenciant le type 1 et 2 est caractéristique d'un changement de milieu (MICHAUD et al., 1988 ; MOUNAIX, 1991 ; LECOMTE-FINIGER, 1994). Cependant les conditions exactes de formation de cette marque restent à définir de façon précise (LECOMTE-FINIGER et al., 1993).

\section{Mélange des flux observés en février}

En février uniquement, les flux sont mélangés, en mer comme en estuaire les trois types d'otolithes et les trois stades pigmentaires sont présents. Ces civelles ont pu pénétré en eau douce avant de repartir en mer car elles sont capturées à proximité d'un courant où elles pénètrent pendant la saison de migration. Le retour en mer après un passage en estuaire est supposé dans des travaux antérieurs basés sur la lecture des otolithes (LECOMTE-FINIGER, 1994). Cette hypothèse d'un retour en mer après un passage en estuaire peut s'expliquer par des conditions environnementales. 
- Hydrodynamisme - Les capacités natatoires des civelles sont limitées en raison du faible développement des fibres musculaires et de la vessie gazeuse qui n'est pas encore fonctionnelle (HICKMAN, 1981). Des tests en conditions expérimentales montrent qu'elles ne peuvent pas lutter contre des courants supérieurs à $0,2-0,3 \mathrm{~m} \cdot \mathrm{s}^{-1}$ (Mc CLEAVE, 1980). De forts débits conjugués à de faibles coefficients de marée empêchent les civelles de poursuivre leur migration en estuaire car les courants ne s'inversent pas pendant le flot (DE CASAMAJOR et al., 2000). Cependant, un apport massif d'eau continentale n'explique pas la présence de civelles aux otolithes de type 2 et 3 en mer, car les débits sont faibles (autour de $150 \mathrm{~m}^{3} . \mathrm{s}^{-1}$ ) pendant et avant les échantillonnages (voir tab.l).

- Température eau douce/eau de mer - Un effet bloquant par différence thermique peut expliquer la présence de civelles aux otolithes de type 2 et 3 en mer en février ainsi que l'absence de différence significative des teneurs en eau et du rapport ADN/ Poids sec entre les deux sites. Une différence thermique supérieures à $3-4^{\circ} \mathrm{C}$ entre les eaux marines et continentales pourrait refouler les civelles de l'estuaire vers la mer (Mc GOVERN et Mc CARTHY, 1992). Dans le bas estuaire, le brassage des eaux limité à quelques kilomètres (DE CASAMAJOR, 1995) ne leur permets pas de franchir le seuil thermique. Cette différence est peu marquée fin novembre $\left(<\right.$ à $\left.3-4^{\circ} \mathrm{C}\right)$ à l'inverse, fin janvier, la température est de $12^{\circ} \mathrm{C}$ en mer, et de $5,5^{\circ} \mathrm{C}$ en estuaire soit un écart de 6 à $7^{\circ} \mathrm{C}$.

\section{Amaigrissement et pertes en eau}

Les variations de la teneur en eau et du rapport ADN/Poids sec entre les échantillons de mer et d'estuaire en décembre résultent de phénomènes d'osmorégulation, pertes en eau et dépenses énergétiques liées à la transition entre le milieu marin et continental (CICCOTTI et al., 1995). L'hyperactivité thyroïdienne incite les civelles à rechercher l'eau moins salée. Elles sont en fin de métamorphose ce qui entraîne une déshydratation qu'elles compensent par leur venue en eau douce (PARRY, 1966 in DURIEU, 1986). Cette adaptation est matérialisée par une double marque en bordure d'otolithe. Les fortes capacités osmotiques des civelles (TOSI et al., 1988 ; CICCOTTI et al., 1993) testées en laboratoire, pourraient expliquer des pertes lipidiques de faible intensité après un jeûne plusieurs mois. Les travaux sur l'hydrodynamisme de l'estuaire de l'Adour (PROUZET et al., 2000) montrent qu'il s'écoule 3 à 4 jours entre la capture en mer et en estuaire ce qui représente un intervalle de temps bref alors que les civelles jeûnent depuis plusieurs semaines. De plus, une faible activité métabolique est signalée sur Anguilla japonica (KAWAKAMI et al., 1999) à partir du rapport ARN/ADN. Des travaux complémentaires, sur le processus d'adaptation des civelles à l'eau douce permettraient de quantifier les dépenses énergétiques en fonction de l'avancée des individus dans l'estuaire et de la diminution de la salinité.

\section{CONCLUSION}

Ce travail précise la phase de transition des civelles du milieu marin à continental. Un amaigrissement des individus est observé en décembre ainsi que des pertes en eau au cours de la traversée de l'estuaire. L'examen des otolithes et de la pigmentation apporte des informations complémentaires. De plus les échantillons de décembre sont constitués d'individus aux caractéristiques homogènes appartenant au même flux. En février, aucune différence n'est observée entre les échantillons de mer et d'estuaire. Un brassage important de ces flux, est observé à partir des stades pigmentaires et des différents types d'otolithes aussi bien en zone côtière qu'en estuaire. L'absence de crue pendant la période d'étude et la forte différence thermique entre les masses d'eau marine et continentale en février explique les résultats obtenus. La comparaison des types d'otolithes et des stades pigmentaires fait apparaître que le stade estuarien $V_{B}$ n'est pas représentatif de l'état de développement physiologique des civelles. Des 
travaux complémentaires permettraient une interprétation plus fine des résultats. Notamment à partir d'un échantillonnage marin éloigné de toute zone d'apport d'eau continentale et d'une meilleure connaissance des conditions de formation de cette double marque en bordure d'otolithe.

\section{REMERCIEMENTS}

Les auteurs remercient l'ensemble des personnes ayant participé à l'échantillonnage biologique. Le personnel du laboratoire d'Ichtyoécologie de l'équipe EPHE-CNRS pour le traitement des échantillons. Les remerciements s'adressent à I'Université de Perpignan et plus particulièrement au laboratoire de Chimie-Physique pour la mise à disposition du spectrofluorimètre et au centre de Microscopie électronique, plus particulièrement Dimitri Gorand pour son aide technique lors des prises de vue avec le MEB.

\section{BIBLIOGRAPHIE}

ANTUNES C., TESCH F.W., 1997. A critical consideration of the metamorphosis zone when identifying daily rings in otoliths of European eel, Anguilla anguilla (L.)., Ecol. Freshwater Fish, 6, 106-107.

ARAI T., OTAKE T., TSUKAMOTO K., 1997. Drastic changes in otolith microstructure and microchemistry accompanying the onset of metamorphosis in the Japanese eel Anguilla japonica. Mar. Ecol. Prog. Ser., 161, 17-22.

ARAI T., OTAKE T., TSUKAMOTO K., 2000. Timing of metamorphosis and larval segregation of the Atlantic eels Anguilla rostrata and Anguilla anguilla as revealed by otolith microstructure and microchemistry. Mar. Biol., 137, 39-45.

ARAI T., LIMBONG D., OTAKE T., TSUKAMOTO K., 1999. Metamorphosis and inshore migration of tropical eels Anguilla spp. In the Indo-Pacific. Mar. Ecol. Prog. Ser., 182, 283-293.

BARBIN G.P., KRUEGER W.H., 1994. Behaviour and swimming performance of elvers of the American eel, Anguilla rostrata, in an experimental flume. J. Fish Biol., 45, 111-121.

BERGERON J.P., BOULHIC M., GALOIS R., 1991. Effet de la privation de la nourriture sur la teneur en adn de la larve de sole (Solea solea L.). J. Mar. Sci. 48, 127-134.

BERGERON J.P., PERSON-LE RUYET J., 1997. Teneur en de la larve de Dicentrarchus labrax : évolution ontogénétique et effet de la privation de nourriture. Aquat. Living Resour., 10, 247-250.

BOËTIUS I., BOËTIUS J., 1989. Ascending elvers, Anguilla anguilla, from five european localities. Analyses of pigmentation stages, condition, chemical composition and energy reserves. Dana, 7, 1-12.

BRU N., 1998. Etude de quelques méthodes d'estimation non-paramétriques de courbes. Application à l'évaluation des flux de civelles d'Anguille.- Thèse Univ. Pau et des Pays de l'Adour, 160p.

CHARLON N., BLANC J.M., 1982. Etude des civelles d'Anguilla anguilla L. dans la région du bassin de l'Adour. 1-Caractéristiques biomètriques de longueur et de poids en fonction de la pigmentation. Arch. Hydrobiol., 93, 238-255.

CICCOTTI E., MACCHI E., ROSSI A., CATALDI E., CATAUDELLA S., 1993. Glass eel (Anguilla anguilla) acclimatation to freshwater and seawater : morphological changes of the digestive tract. J. appl. Ichtyol., 9, 74-81.

CICCOTTI E., RICCI T., SCARDI M., FRESI E. CATAUDELLA S., 1995. Interseasonal characterization of glass eel migration in the River Tiber : space and time dynamics.- $J$. Fish Biol., 47, 248-255.

DAEMEN E, VOLCKAERT F.A.M., CROSS T et OLLEVIER F., 1999. New evidence of genetic structure in european eel. EIFAC/ICES, Working group on eel, Silkeborg, Denmark 20-24/09/1999. 
DE CASAMAJOR M.N., 1995. Mesures de salinité et température sur l'estuaire de l'Adour.Rapport interne IFREMER, 37p.

DE CASAMAJOR M.-N., 1998. Comportement migratoire de la civelle d'anguille (Anguilla anguilla L.) dans l'estuaire de l'Adour en fonction de la variabilité des conditions environnementales. Thèse Univ. Pau et des Pays de l'Adour, 138p + Annexes.

DE CASAMAJOR M.-N., BRU N., PROUZET P., 1999. Influence de la luminosité nocturne et de la turbidité sur le comportement vertical de migration de la civelle d'Anguille (Anguilla anguilla L.) dans l'estuaire de l'Adour.- Bull. Fr. Pêche Piscic., 355, 327347.

DE CASAMAJOR M.-N, PROUZET P., LAZURE P., 2000. Identification des flux de civelle (Anguilla anguilla) à partir des relations d'allométrie en fonction des conditions hydrodynamiques dans l'estuaire de l'Adour. Aquat.Living. Resour. 13, 411-420.

DE CASAMAJOR M.-N., LECOMTE-FINIGER R., PROUZET P., 2001. Détermination de l'état d'amaigrissement des civelles (Anguilla anguilla) en migration en zone côtière et estuarienne. C. R. Acad. Sc., Sous-presse.

DESAUNAY Y., GUERAULT D., 1997. Seasonal and long-term changes in biometrics of eel larvae : a possible relationship between recruitment variation and North Atlantic ecosystem productivity. J. Fish Biol. 51, 317-339.

DESAUNAY Y., LECOMTE-FINIGER R., GUÉRAULT D., 1996, Mean age and migration patterns of Anguilla anguilla glass eels from three french estuaries (Somme, Vilaine et Adour rivers). Arch. Pol. Fish. 4, 187-194.

DURRIEU L., 1986.- Contribution à l'étude de la Civelle en estuaire de Loire.- Thèse Doc. Vétérinaire, Univ. de Nantes, 98p.

ELIE P., LECOMTE-FINIGER R., CANTRELLE I., CHARLON N., 1982. Définition des limites des différents stades pigmentaires durant la phase civelle d'Anguilla anguilla L. (Poisson Téléostéen Anguilliforme). Vie Milieu, 32, 149-157

GUERAULT D., LECOMTE-FINIGER R., DESAUNAY Y., BIAGIANTI-RISBOURG S., BEILLOIS P., GRELLIER P., 1992. Glass eel arrivals in the Vilaine estuary (Northern Bay of Biscay) in 1990 : Demographic features and early life history.- Irish Fisheries Investigations Serie A (Freshwater), 36, 5-14.

HARO A.J., KRUEGER W.H., 1987. Pigmentation, size and migration of elvers (Anguilla rostrata (Lesueur)) in a coastal Rhode Island stream. Can. J. Zool., 66, 2528-2533.

HICKMAN R.A., 1981. Densities and swimbladder development of juvenile American eels, Anguilla rostrata (Lesueur) as related to energetics of migration. J. Fish Biol., 18, 507-517.

KARSTEN U., WOLLENBERGER A., 1972. Determination of DNA and RNA in homogenised cells and tissues by surface fluorometry. Anal. Biochem., 46, 135-148.

KARSTEN U., WOLLENBERGER A., 1977. Improvements in the ethidium bromide method for direct fluorimetric estimation of DNA and RNA in cell and tissue homogenates. Anal. Biochem. 77, 464-470.

KAWAKAMI Y., MOCHIOKA N. KIMURA R., NAKAZONO A., 1999. Seasonal changes of the $\mathrm{rna} / \mathrm{dna}$ ratio, size and lipid contents and immigration adaptability of japonese glass-eels, Anguilla japonica, collected in northern Kyushu, Japan. J. Exp. Mar. Biol. Ecol., 238, 1-19.

LECOMTE-FINIGER R., 1983. Etude morphométrique et énergétique de la civelle de méditerranée au cours de la pigmentation. Vie et Milieu, 33, 87-92.

LECOMTE-FINIGER R., 1994. Contribution de l'otolithométrie à l'étude de la dynamique de la migration larvaire de l'anguille européenne Anguilla anguilla. Bull. Fr. Pêche Piscic., 335, 17-31.

LECOMTE-FINIGER R., 1992. Growth history and age at recruitment of European eels (Anguilla anguilla) as revealed by otolith microstructure. Mar. Biol., 114, 205-210.

LECOMTE-FINIGER R., YAHYAOUI A., 1989. La microstructure de l'otolithe au service de la connaissance du développement larvaire de l'anguille européenne Anguilla anguilla. C.R. Acad. Sci. Paris, 308 (Serie III), 1-7. 
LECOMTE-FINIGER R., DESAUNAY Y., GUÉRAULT D., GRELLIER P., 1993. The immigration of Anguilla anguilla (L.) glass eels in coastal waters : questions about the determinism of the otolith structures. $8^{\text {th }}$ session of the eifac, Working Party of Eel, 3p.

LEE T.W., LEE K.S., 1989. Daily growth increments and lunar pattern in otolith of the eel, Anguilla japonica, in the freshwater. Bull. Korean Fish. Soc., 22, 36-40.

LE PECQ J.B., PAOLETTI C., 1966. A new fluorometric method for RNA and DNA determination. Analytical biochemistry, 17, 100-107.

Mc CLEAVE J.D., 1980. Swimming performance of European eel (Anguilla anguilla (L.)) elvers. J. Fish. Biol., 16, 445-452.

Mc GOVERN P., Mc CARTHY T.K., 1992. Elvers migration in the River Corrib system, western Ireland. Irish Fisheries Investigations Series A (Freshwater), 36, 25-32.

MICHAUD M., DUTIL J.-D., DODSON J.J., 1988. Determination of young American eels, Anguilla rostrata, in fresh water, based on otolith surface area and microstructure. J. Fish Biol., 32, 179-189.

MOUNAIX B., 1991, Validation de l'estimation de l'age de l'anguille européenne, Anguilla anguilla, dans le bassin versant de la Vilaine (Bretagne) : résultats préliminaires. Otolithométrie, 109-117.

PANNELLA G., 1971, Fish otoliths : daily growth layers and periodical patterns. Science (Wash., D.C.), 173, 1124-1127.

PROUZET P., SANCHEZ F., LAZURE P., 2000. Modèle hydrodynamique à une dimension de l'estuaire de l'Adour : mise au point et calibration, application à la modélisation de la migration de la civelle dans l'estuaire. Rap. Ifremer, 25p. + annexes

TOSI L., SALA L., SOLA C., SPAMPANATO A., TONGIORGI. P., 1988. Experimental analysis of the thermal and salinity preferences of glass-eels, Anguilla anguilla (L.) before and during the upstream migration. J. Fish Biol., 33, 721-733.

STRUBBERG A., 1913. The metamorphosis of elvers is influenced by outward conditions some experiments. Meddelelser Fra Kommissionen for Havundersogelser, 3, 11p.

UMEZAWA A., TSUKAMOTO K., 1991. Factors influencing otolith increment formation in Japanese eel, Anguilla japonica T. et S., elvers. J. Fish Biol., 39, 211-223.

WILCOXON F., 1945. Individual comparisons by ranking methods. Biometrics, 1, 80-83. 
